\title{
A GENERALISATION OF THE MATROID LIFT CONSTRUCTION
}

\author{
GEOFF WHITTLE
}

\begin{abstract}
This paper introduces a general matroid-theoretic construction which includes, as special cases, elementary lifts of matroids and bias matroids of biased graphs. To perform the construction on a matroid $M$, it is necessary (but not sufficient) to have a submodular function inducing $M$. Elementary lifts are obtained when the submodular function chosen is the rank function of $M$.

We define what is meant by a $k$-induced matroid. These matroids simultaneously generalise matroids of graphs, transversal matroids and Dilworth truncations. They are induced by a particularly natural class of submodular functions. The effect of the above construction on $k$-induced matroids using these natural submodular functions is studied. Results on minors of $k$-induced matroids and the matroids obtained from them using the construction are given.
\end{abstract}

\section{INTRODUCTION}

A set $\mathscr{C}$ of circuits of a matroid $M$ is a linear class if whenever $C_{1}$ and $C_{2}$ are a modular pair of circuits both belonging to $\mathscr{C}$, then any circuit of $M$ contained in $C_{1} \cup C_{2}$ also belongs to $\mathscr{C}$. Linear classes of circuits are important in matroid theory since they induce elementary lifts of matroids (see, for example, [24, §3]).

What is striking is that in certain circumstances linear classes of circuits can be used to obtain another construction. As part of an important series of papers [23-28], Zaslavsky shows that a linear class of circuits of a graph can be used to construct a matroid on the edges of the graph; the so-called bias matroid. It is evident from the most casual reading of Zaslavsky's papers that such bias matroids form a significant class. For example, Dowling group geometries are bias matroids. (Dowling group geometries are defined and studied in [8]. Further properties of this interesting class of matroids can be found in [7, 11, 21].) If $\mathscr{B}$ is a linear class of circuits of a graph then, typically, the bias matroid constructed using $\mathscr{B}$ is not isomorphic to the elementary lift of the cycle matroid of the graph obtained via $\mathscr{B}$. That is, a linear class of circuits of a graph can be used for two distinct constructions.

What is happening here? This paper provides an answer by showing that bias matroids and matroid lifts are but two instances of a more general matroid construction. To perform the construction it is necessary to have a linear class of

Received by the editors March 21, 1988.

1980 Mathematics Subject Classification (1985 Revision). Primary 05B35.

(C) 1989 American Mathematical Society $0002-9947 / 89 \$ 1.00+\$ .25$ per page 
circuits of a matroid. But this is not sufficient; an increasing, submodular function inducing the matroid is also needed. The key result of this paper (Theorem 3.4) describes this matroid construction. The matroid lift construction, for example, corresponds to choosing the rank function of $M$ (which is an increasing submodular function). On the other hand, bias matroids of graphs arise from a different choice of submodular function (namely the function whose value on a subset $E^{\prime}$ of edges of a graph is one less than the cardinality of the subset of vertices incident with edges in $E^{\prime}$ ).

The paper is organised as follows. $\S 2$ consists of basic results on submodular functions. In $\S 3$, the theorem discussed above is presented. $\S 4$ considers a class of matroids, called $k$-induced matroids. These matroids provide a natural and common generalisation of such important classes as graphic matroids, transversal matroids and Dilworth truncations. They are of particular interest in this paper because they are most naturally defined via a submodular function which is, typically, distinct from their rank function. $\S 5$ considers bias matroids and $\S 6$ presents results on minors of $k$-induced matroids and minors of matroids obtained from $k$-induced matroids via the construction of Theorem 3.4.

The theory in this paper has a number of applications. For example, it is possible to use the theory to construct generalised Dowling group geometries (a construction anticipated by Mason [13]). Here one begins with an arbitrary matroid $M$ and a finite group $A$. A matroid is then constructed in which the dependencies are determined by both the matroid structure in $M$ and group multiplication in $A$. The usual Dowling group geometries are obtained when $M$ is a free matroid. These results will appear elsewhere.

Another possible application is in the theory of weak maps. To some extent this study is initiated in [24].

We assume that the reader is familiar with the basic concepts of matroids as set forth in [19, 20]. Notation will in general follow [19] with some minor differences as follows. Let $M$ be a matroid with ground set $E$; we say that $M$ is a matroid on $E$. For a subset $S \subseteq E$, the restriction of $M$ to $E \backslash S$ will be denoted by $M \mid(E \backslash S)$ or by $M \backslash S$ according to convenience, and the contraction of $M$ to $E \backslash S$ will be denoted by $M / S$. The closure and rank of $S$ in $M$ will be denoted by $\mathrm{cl}_{M}(S)$ and $r_{M}(S)$, respectively, or if no danger of ambiguity exists by $\mathrm{cl}(S)$ and $r(S)$ respectively.

All matroids are assumed to have finite ground sets.

\section{Submodular functions}

A function $\mu$ from the power set of a set $E$ into the integers is increasing if whenever $A$ and $B$ are subsets of $E$ with $A \subseteq B$, then $\mu(A) \leq \mu(B)$. It is submodular if $\mu(A)+\mu(B) \geq \mu(A \cap B)+\mu(A \cup B)$ for all subsets $A$ and $B$ of E. 
Now for any increasing submodular function $\mu: 2^{E} \rightarrow Z$ we have

Proposition 2.1. The subsets $I \subseteq E$ such that $\left|I^{\prime}\right| \leq \mu\left(I^{\prime}\right)$ for all nonempty subsets $I^{\prime} \subseteq I$ are the independent sets of a matroid on $E$.

This proposition is well known. For example it appears in Crapo and Rota [5, Proposition 7.3] and Pym and Perfect [16, Corollary 4.4]. The matroid obtained via Proposition 2.1 is the matroid induced by $\mu$. An immediate corollary of Proposition 2.1 is

Corollary 2.2. The circuits of the matroid induced by $\mu$ are the minimal nonempty subsets $C \subseteq E$ with the property that $|C|>\mu(C)$.

If $M$ is a matroid on $E$ induced by an increasing submodular function $\mu$, then a subset $A \subseteq E$ is $\mu$-normal if $r_{M}(A)=\mu(A)$. One would expect that subsets on which the value of the submodular function is equal to their rank are of interest and this turns out to be the case. The above definition agrees with that of Crapo and Rota [5, Chapter 7] when $A$ is independent. Dawson [6] and Lintzeris [12] call $\mu$-normal subsets $\mu$-balanced but we reserve "balanced" for another use. Crapo and Rota restrict their discussion of these sets to submodular functions which take the value one on singletons. Dawson and Lintzeris restrict theirs to submodular functions which take the value zero on the empty set. Neither of these restrictions are in place in this paper. In fact some of our most useful examples of submodular functions neither take the value one on all singletons nor take the value zero on the empty set. The following results are essentially weakened versions of similar results of the above authors. The weakening is necessary. A result of Crapo and Rota [5, Proposition 7.4], namely that an independent set can be partitioned into its maximal $\mu$-normal subsets, does not always hold for submodular functions which do not necessarily take the value one on singletons. (For example an independent singleton may not be $\mu$-normal.) It will presently be seen that a result of Dawson [6, Theorem 5 (ii)], namely that a union of $\mu$-normal sets is $\mu$-normal, does not hold for submodular functions which may take negative values.

In the following propositions $M$ is a matroid on $E$ induced by the increasing submodular function $\mu$.

Proposition 2.3. If $A \subseteq E$ with $r_{M}(A)>0$, then $\mu(A) \geq r_{M}(A)$. In particular, if $A$ is a nonempty loopless subset of $E$ then $\mu(A)>0$.

Proof. If $I$ is a maximal independent set of $M$ contained in $A$, then $\mu(A) \geq$ $\mu(I) \geq|I|=r_{M}(A)>0$.

A broken circuit of a matroid is a set of the form $C \backslash x$ for some circuit $C$ of $M$ and $x \in C$. Usually the term "broken circuit" denotes a set of the form $C \backslash x$ where $x$ is the largest element of $C$ in some fixed linear ordering of $E$. Since, here, $E$ has no linear order imposed on it, no danger of confusion should arise. 
Proposition 2.4. (i) All circuits which are not loops are $\mu$-normal.

(ii) Nonempty broken circuits of $M$ are $\mu$-normal in $M$.

(iii) If $I_{1}$ and $I_{2}$ are $\mu$-normal in $M, I_{1} \cup I_{2}$ is independent in $M$ and $I_{1} \cap I_{2}$ is nonempty, then $I_{1} \cap I_{2}$ and $I_{1} \cup I_{2}$ are both $\mu$-normal in $M$.

Proof. Let $C$ be a circuit of $M$ which is not a loop. Then $|C|>1$, so by Corollary 2.2, $\mu(C)<|C|$ and $\mu(C) \leq r_{M}(C)$. Now $C$ is not a loop so by Proposition 2.3, $\mu(C) \geq r_{M}(C)$. Therefore $\mu(C)=r_{M}(C)$ and (i) is established. Now if $x$ belongs to $C$ then $C \backslash x$ is a nonempty broken circuit of $M$. Using Proposition 2.3 we see that $\mu(C \backslash x) \geq r_{M}(C \backslash x)=r_{M}(C)=\mu(C)$. But $\mu$ is increasing so $\mu(C) \geq \mu(C \backslash x)$. Therefore the above inequality is an equality and (ii) is established.

If $I_{1}$ and $I_{2}$ are $\mu$-normal, $I_{1} \cup I_{2}$ is independent and $I_{1} \cap I_{2}$ is nonempty, then all these sets are nonempty and independent and it is easily seen that $\mu\left(I_{1}\right)=\left|I_{1}\right|, \mu\left(I_{2}\right)=\left|I_{2}\right|, \mu\left(I_{1} \cup I_{2}\right) \geq\left|I_{1} \cup I_{2}\right|$ and $\mu\left(I_{1} \cap I_{2}\right) \geq\left|I_{1} \cap I_{2}\right|$. Now

$$
\begin{aligned}
\left|I_{1}\right|+\left|I_{2}\right| & =\mu\left(I_{1}\right)+\mu\left(I_{2}\right) \geq \mu\left(I_{1} \cup I_{2}\right)+\mu\left(I_{1} \cap I_{2}\right) \\
& \geq\left|I_{1} \cup I_{2}\right|+\left|I_{1} \cap I_{2}\right|=\left|I_{1}\right|+\left|I_{2}\right| .
\end{aligned}
$$

Therefore all of the above inequalities are equalities. This is only possible if both $I_{1} \cup I_{2}$ and $I_{1} \cap I_{2}$ are $\mu$-normal and (iii) is established.

Proposition 2.5. If $A \subseteq E$ then $\mu(A)=\mu\left(\mathrm{cl}_{M}(A)\right)$.

Proof. We show that if $x \in \operatorname{cl}_{M}(A)$ then $\mu(A)=\mu(A \cup x)$. If $x \in A$ then this is trivial so assume that $x \notin A$. In this case, there exists an independent subset $I \subseteq A$ such that $I \cup x$ is a circuit of $M$. By Proposition 2.4(i) and (ii), $\mu(I)=\mu(I \cup x)$. This fact, the submodularity of $\mu$ and elementary set theory show that

$$
\begin{aligned}
\mu(A \cup x) & =\mu((I \cup x) \cup A) \\
& \leq \mu(I \cup x)+\mu(A)-\mu((I \cup x) \cap A) \\
& =\mu(I)+\mu(A)-\mu(I)=\mu(A) .
\end{aligned}
$$

The result follows routinely from the above observation.

Proposition 2.6. If $A$ is connected in $M$ with $r_{M}(A)>1$ then $A$ is $\mu$-normal in $M$.

Proof. Assume that $A$ is connected in $M$ with $r_{M}(A)>1$. Let $B$ be a basis of $M \mid A$ and let $B^{\prime}$ be a maximal $\mu$-normal subset of $B$. For $a \in A \backslash B$, let $B_{a}$ denote the subset of $B$ such that $B_{a} \cup\{a\}$ is a circuit of $M$ (that is, such that $B_{a} \cup\{a\}$ is the basic circuit of $\{a\}$ with respect to the basis $B$ ). Since $r_{M}(A)>1, A \backslash B$ is nonempty and since $A$ is loopless (otherwise $A$ is not connected), $B_{a} \neq \varnothing$ for any $a \in A \backslash B$. Now $B_{a}$ is a nonempty broken circuit of $M$ so by Proposition 2.4(ii), $B_{a}$ is $\mu$-normal. Therefore $B^{\prime} \neq \varnothing$. If $B_{a} \cap B^{\prime} \neq \varnothing$, then by Proposition 2.4(iii), $B_{a} \cup B^{\prime}$ is $\mu$-normal so $B_{a} \subseteq B^{\prime}$. That is, if $a \in A \backslash B$ then either $B_{a} \subseteq B^{\prime}$ (in which case $\{a\}$ is contained in $\mathrm{cl}_{M \mid A}\left(B^{\prime}\right)$ ) or $B_{a} \subseteq B \backslash B^{\prime}$ (in which case $\{a\}$ is contained in $\mathrm{cl}_{M \mid A}\left(B \backslash B^{\prime}\right)$ ). 
Therefore $\mathrm{cl}_{M \mid A}\left(B^{\prime}\right)$ and $\mathrm{cl}_{M \mid A}\left(B \backslash B^{\prime}\right)$ partition $A$. But $r_{M}(A)=\left|B^{\prime}\right|+\left|B \backslash B^{\prime}\right|$. Since $M \mid A$ is connected this situation is only possible if $B \backslash B^{\prime}=\varnothing$; that is if $B$ is $\mu$-normal. But by Proposition $2.5 \mu(A)=\mu(B)$ so $A$ is $\mu$-normal.

We illustrate the significance of $\mu$-normal subsets with an example. Let $G=$ $(V, E)$ be a graph. For a subset $E^{\prime} \subseteq E$ let $V\left(E^{\prime}\right)$ denote the set of vertices of $G$ which are incident with edges in $E^{\prime}$. Then the function $\mu: 2^{E} \rightarrow Z$ defined by $\mu\left(E^{\prime} j=\left|V\left(E^{\prime}\right)\right|-1\right.$ is a submodular function inducing $M(G)$, the cycle matroid of $G$. (This is well known; see, for example, Welsh [19, Chapter 8]. It is also a consequence of later results in this paper.) Now $E^{\prime}$ is $\mu$-normal if $r_{M(G)}\left(E^{\prime}\right)=\left|V\left(E^{\prime}\right)\right|-1$; that is, if the number of vertices of $G$ incident with edges in $E^{\prime}$ is one greater than the rank of the cycle matroid of the subgraph of $G$ induced by $E^{\prime}$. But this is precisely the condition for this subgraph to contain a spanning tree and therefore to be connected (as a graph). In this case $\mu$-normality just corresponds to graph connectedness and Proposition 2.4 and 2.6 give some (rather unsurprising) results about connected sets in graphs. Note that the union of two vertex disjoint circuits in a graph is not connected. That is, a union of $\mu$-normal subsets is not necessarily $\mu$-normal.

\section{3. $\mu$-LIFTS}

Let $M$ and $M^{\prime}$ be matroids sharing a common ground set. If every flat of $M$ is a flat of $M^{\prime}$ then $M$ is a quotient of $M^{\prime}$ and $M^{\prime}$ is a lift of $M$. If it is also the case that $r\left(M^{\prime}\right) \leq r(M)+1$, then $M$ is an elementary quotient of $M^{\prime}$ and $M^{\prime}$ is an elementary lift of $M$. Note that alternative (but intimately related) definitions of "lift" have been given elsewhere [13, 15].

Let $\mathscr{B}$ be a set of circuits of a matroid $M$; recall that $\mathscr{B}$ is a linear class if whenever $C_{1}$ and $C_{2}$ are a modular pair of circuits both of which belong to $\mathscr{B}$, then any circuit $C$ of $M$ contained in $C_{1} \cup C_{2}$ belongs to $\mathscr{B}$. (Recall also that $A$ and $B$ are a modular pair of subsets of $M$ if $r(A)+r(B)=r(A \cup B)+r(A \cap B)$.) If $\mathscr{B}$ is a linear class of circuits of the matroid $M$ on $E$, then a subset $S \subseteq E$ is $\mathscr{B}$-balanced if every circuit of $M$ contained in $S$ belongs to $\mathscr{B}$. It follows trivially that all independent sets are $\mathscr{B}$-balanced.

One reason for interest in linear classes of circuits is that they determine elementary lifts. The following proposition is a routine consequence of Brylawski [3, Proposition 7.4.15]. Zaslavsky [24, §3] is also of interest in this context.

Proposition 3.1. Let $M$ be a matroid on $E$ and $\mathscr{B}$ be a linear class of circuits of $M$. Then the function $r_{M^{\prime}}: 2^{E} \rightarrow Z$ defined by

$$
r_{M^{\prime}}(A)= \begin{cases}r_{M}(A) & \text { if } A \text { is } \mathscr{B} \text {-balanced, } \\ r_{M}(A)+1 & \text { if } A \text { is not } \mathscr{B} \text {-balanced }\end{cases}
$$

for all subsets $A \subseteq E$ is the rank function of an elementary lift $M^{\prime}$ of $M$. Furthermore any elementary lift of $M$ can be obtained in such a way.

The matroid $M^{\prime}$ defined by Proposition 3.1 is the lift of $M$ induced by $\mathscr{B}$. 
The main result of this section (Theorem 3.4) shows that $M^{\prime}$ is not necessarily the only matroid "induced" by $\mathscr{B}$. Other matroids may arise by choosing appropriate submodular functions inducing $M$. The following alternative characterisation of modular pairs of circuits shall prove useful.

Proposition 3.2. Distinct circuits $C_{1}$ and $C_{2}$ of a matroid $M$ are a modular pair of circuits of $M$ if and only if $\left|C_{1} \cup C_{2}\right|=r\left(C_{1}\left(\mid C_{2}\right)+2\right.$.

Proof. Since $C_{1}$ and $C_{2}$ are distinct, $C_{1} \cap C_{2}$ is independent in $M$ so $\left|C_{1} \cap C_{2}\right|=r\left(C_{1} \cap C_{2}\right)$ and then $\left|C_{1} \cup C_{2}\right|=\left|C_{1}\right|+\left|C_{2}\right|-\left|C_{1} \cap C_{2}\right|=r\left(C_{1}\right)+r\left(C_{2}\right)-$ $r\left(C_{1} \cap C_{2}\right)+2$. The result follows routinely from an examination of this equation.

Let $\mu: 2^{E} \rightarrow Z$ be an increasing submodular function and define $(\mu+1)$ : $2^{E} \rightarrow Z$ by $(\mu+1)\left(E^{\prime}\right)=\mu\left(E^{\prime}\right)+1$ for all subsets $E^{\prime} \subseteq E$. As is well known (see, for example, Crapo and Rota [5, Proposition 7.1]), $\mu+1$ is also increasing and submodular. If $M$ is the matroid on $E$ induced by $\mu$ and $N$ is the matroid on $E$ induced by $\mu+1$ then it follows immediately from the definition that $M$ is a weak map image of $N$. (Recall that the matroid $M_{1}$ is a weak map image of the matroid $M_{2}$ if both share a common ground set and every independent set of $M_{1}$ is independent in $M_{2}$.)

The following lemma is routine.

Lemma 3.3. If $C$ is a circuit of $M$ which is not a loop, then $C$ is a $(\mu+1)$ normal independent set in $N$.

Theorem 3.4. Let $E$ be a set and $\mu: 2^{E} \rightarrow Z$ be an increasing, submodular function. Let $M$ and $N$ be the matroids on $E$ induced by $\mu$ and $\mu+1$ respectively. Let $\mathscr{B}$ be a linear class of circuits of $M$ and $\mathscr{C}^{\prime}$ be the set of circuits of $N$ which contain no member of $\mathscr{B}$. Then $\mathscr{C}^{\prime} \cup \mathscr{B}$ is the set of circuits of a matroid on $E$.

Proof. If $C_{1}$ and $C_{2}$ belong to $\mathscr{C}^{\prime} \cup \mathscr{B}$, then it is only plausible that $C_{2}$ properly contains $C_{1}$ if $C_{2} \in \mathscr{B}$ and $C_{1} \in \mathscr{C}^{\prime}$. But by Lemma 3.3, $C_{2}$ is independent in $N$ so this situation cannot occur.

Now assume that $C_{1}$ and $C_{2}$ are distinct members of $\mathscr{C}^{\prime} \cup \mathscr{B}$ and that $x \in C_{1} \cap C_{2}$. We must show that $\left(C_{1} \cup C_{2}\right) \backslash x$ contains a member of $\mathscr{C}^{\prime} \cup \mathscr{B}$.

If $C_{1}$ and $C_{2}$ are both members of $\mathscr{C}^{\prime}$, then $\left(C_{1} \cup C_{2}\right) \backslash x$ contains a circuit $C$ of $N$. Either $C \in \mathscr{C}^{\prime}$ or $C$ contains a member of $\mathscr{B}$. In either case $\left(C_{1} \cup C_{2}\right) \backslash x$ contains a member of $\mathscr{C}^{\prime} \cup \mathscr{B}$.

Assume that $C_{1}$ and $C_{2}$ are both members of $\mathscr{B}$. If $C_{1}$ and $C_{2}$ are a modular pair in $M$, then $\left(C_{1} \cup C_{2}\right) \backslash x$ contains a member of $\mathscr{B}$ so assume that $C_{1}$ and $C_{2}$ are not a modular pair. In this case, by Proposition 3.2, $r_{M}\left(C_{1} \cup C_{2}\right)<\left|C_{1} \cup C_{2}\right|-2$. Therefore $r_{M}\left(\left(C_{1} \cup C_{2}\right) \backslash x\right)<\left|\left(C_{1} \cup C_{2}\right) \backslash x\right|-1$. Now $C_{1} \cup C_{2}$ is connected in $M$ and $r_{M}\left(C_{1} \cup C_{2}\right)>1$. (Neither $C_{1}$ nor $C_{2}$ can be loops of $M$-a loop cannot have nontrivial intersection with another circuit-so $r_{M}\left(C_{1} \cup C_{2}\right) \geq 1$. If $r_{M}\left(C_{1} \cup C_{2}\right)=1$ then $C_{1}$ and $C_{2}$ must form 
a modular pair in $M)$. Therefore $C_{1} \cup C_{2}$ is $\mu$-normal by Proposition 2.6. But $x \in \operatorname{cl}\left(\left(C_{1} \cup C_{2}\right) \backslash x\right)$ so $\left(C_{1} \cup C_{2}\right) \backslash x$ must also be $\mu$-normal by Proposition 2.5. That is, $\mu\left(\left(C_{1} \cup C_{2}\right) \backslash x\right)=r_{M}\left(\left(C_{1} \cup C_{2}\right) \backslash x\right)$. But since $r_{M}\left(\left(C_{1} \cup C_{2}\right) \backslash x\right)<$ $\left|\left(C_{1} \cup C_{2}\right) \backslash x\right|-1$ it follows that $\mu\left(\left(C_{1} \cup C_{2}\right) \backslash x\right)<\left|\left(C_{1} \cup C_{2}\right) \backslash x\right|-1$. That is, $(\mu+1)\left(\left(C_{1} \cup C_{2}\right) \backslash x\right)<\left|\left(C_{1} \cup C_{2}\right) \backslash x\right|$. Therefore $\left(C_{1} \cup C_{2}\right) \backslash x$ is dependent in $N$ and contains a circuit of $N$. As before this guarantees that $\left(C_{1} \cup C_{2}\right) \backslash x$ contains a member of $\mathscr{B} \cup \mathscr{C}^{\prime}$.

Now assume that $C_{1} \in \mathscr{C}^{\prime}$ and $C_{2} \in \mathscr{B}$. By Proposition 2.4(i) and Lemma 3.3, $C_{1}$ is $(\mu+1)$-normal in $N$ and $C_{2}$ is a $(\mu+1)$-normal independent set in $N$. Therefore $(\mu+1)\left(C_{1}\right)=\left|C_{1}\right|-1$ and $(\mu+1)\left(C_{2}\right)=\left|C_{2}\right|$. Now $C_{1} \cap C_{2}$ is a proper subset of $C_{2}$ so $C_{1} \cap C_{2}$ is independent in $M$. So $\mu\left(C_{1} \cap C_{2}\right) \geq\left|C_{1} \cap C_{2}\right|$ and hence $(\mu+1)\left(C_{1} \cap C_{2}\right)>\left|C_{1} \cap C_{2}\right|$. But

$$
(\mu+1)\left(C_{1} \cup C_{2}\right) \leq(\mu+1)\left(C_{1}\right)+(\mu+1)\left(C_{2}\right)-(\mu+1)\left(C_{1} \cap C_{2}\right)
$$

so

$$
(\mu+1)\left(C_{1} \cup C_{2}\right)<\left|C_{1}\right|-1+\left|C_{2}\right|-\left|C_{1} \cap C_{2}\right|=\left|C_{1} \cup C_{2}\right|-1 .
$$

Therefore $(\mu+1)\left(\left(C_{1} \cup C_{2}\right) \backslash x\right)<\left|\left(C_{1} \cup C_{2}\right) \backslash x\right|$ and $\left(C_{1} \cup C_{2}\right) \backslash x$ is dependent in $N$ and thus contains a member of $\mathscr{C}^{\prime} \cup \mathscr{B}$.

In all cases $\left(C_{1} \cup C_{2}\right) \backslash x$ contains a member of $\mathscr{C}^{\prime} \cup \mathscr{B}$ and the theorem is proved. $\mathscr{B}$.

The matroid constructed in the above theorem is the $\mu$-lift of $M$ induced by

Of course one submodular function which induces a matroid $M$ is its rank function $r$. It therefore makes sense to talk about $r$-lifts of $M$. It is not surprising that $r$-lifts are just elementary lifts.

Proposition 3.5. Let $M$ be a matroid on $E$ with rank function $r$ and let $\mathscr{B}$ be a linear class of circuits of $M$. Then the elementary lift of $M$ induced by $\mathscr{B}$ is equal to the r-lift of $M$ induced by $\mathscr{B}$.

Proof. Let $N$ be the matroid on $E$ induced by $r+1$ and let $\mathscr{C}^{\prime}$ be the set of circuits of $N$ which contain no member of $\mathscr{B}$. Then $\mathscr{C}^{\prime} \cup \mathscr{B}$ is the set of circuits of the $r$-lift of $M$ induced by $\mathscr{B}$. We show that $\mathscr{C}^{\prime} \cup \mathscr{B}$ is the set of circuits of the elementary lift of $M$ induced by $\mathscr{B}$. Denote this elementary lift by $M^{\prime}$.

Assume that $C \in \mathscr{C}^{\prime} \cup \mathscr{B}$. If $C \in \mathscr{B}$ then all subsets of $C$ are $\mathscr{B}$ balanced so by Proposition 2.1, $r_{M^{\prime}}\left(C^{\prime}\right)=r_{M}\left(C^{\prime}\right)$ for all subsets $C^{\prime} \subseteq C$ and $C$ is therefore a circuit of $M^{\prime}$. If $C \in \mathscr{C}^{\prime}$, then $C$ is a minimal subset of $E$ with $(r+1)(C)<|C|$; that is $r_{M}(C)<|C|-1$. Now $C$ is dependent in $M$ but contains no member of $\mathscr{B}$ so $C$ is not $\mathscr{B}$-balanced. Therefore $r_{M^{\prime}}(C)=r_{M}(C)+1<|C|$ and $C$ is dependent in $M^{\prime}$. Let $C^{\prime}$ be a proper subset of $C$; then $(r+1)\left(C^{\prime}\right)=r_{M}\left(C^{\prime}\right)+1>\left|C^{\prime}\right|$. If $C^{\prime}$ is independent in $M$, then $C^{\prime}$ is certainly independent in $M^{\prime}$. If $C^{\prime}$ is dependent in $M$, then 
$C^{\prime}$ is not $\mathscr{B}$-balanced so $r_{M^{\prime}}\left(C^{\prime}\right)=r_{M}\left(C^{\prime}\right)+1 \geq\left|C^{\prime}\right|$ and $C^{\prime}$ is independent in $M^{\prime}$. Therefore $C$ is a circuit of $M^{\prime}$.

Assume that $C$ is a circuit of $M^{\prime}$. If $C$ is $\mathscr{B}$-balanced then $C \in \mathscr{B}$. If $C$ is not $\mathscr{B}$-balanced then $r_{M^{\prime}}(C)=r_{M}(C)+1=|C|-1$. That is, $(r+1)(C)<|C|$ and $C$ is therefore dependent in $N$. Let $C^{\prime}$ be a proper subset of $C$. If $C^{\prime}$ is independent in $M$, then $C^{\prime}$ is independent in $N$. If $C^{\prime}$ is dependent in $M$, then $C^{\prime}$ is not $\mathscr{B}$-balanced so $r_{M^{\prime}}\left(C^{\prime}\right)=r_{M}\left(C^{\prime}\right)+1$. But $r_{M^{\prime}}\left(C^{\prime}\right) \geq\left|C^{\prime}\right|$. Therefore $(r+1)\left(C^{\prime}\right) \geq\left|C^{\prime}\right|$. It follows that all proper subsets of $C$ are independent in $N$ and therefore $C$ is a circuit of $N$. But since $C$ is not $\mathscr{B}$-balanced, $C$ contains no member of $\mathscr{B}$. So $C \in \mathscr{C}^{\prime}$ and the proposition is proved.

It follows from Proposition 3.5 that $\mu$-lifts are indeed generalisations of elementary lifts. That the generalisation is nontrivial is demonstrated in $\S 5$. But note that it is frequently the case that the $\mu$-lift of $M$ induced by $\mathscr{B}$ may be equal to the elementary lift induced by $\mathscr{B}$ even when $\mu$ is not the rank function of $M$. In general a matroid is induced by many submodular functions apart from the rank function. Heuristically, there seems to be no good reason to concentrate exclusively on the rank function in the development of structure theory for matroids.

For the remainder of this paper attention is focused on, and Theorem 3.4 is applied to, matroids induced by a particularly natural class of submodular functions. We consider these now.

\section{4. $k$-INDUCED MATROIDS}

First some terminology. Let $Q$ be a matroid on a set $S, E$ be a finite set and $\psi$ be a function from $E$ into the power set of $S$. That is, $(\psi(e), e \in$ $E$ ) is a family of subsets of $S$. For want of a better name we say that the ordered triple $(Q, E, \psi)$ is a hypergraph on $Q$. It may well be the case that $\bigcup_{e \in E} \psi(e) \neq S$ and that $\psi(e)=\varnothing$ for some $e \in E$ so that restrictions on hypergraphs demanded by some authors (for example, Berge [1, Chapter 17]) are not in place here.

We wish to use dependence in $Q$ to define a matroid on $E$ (in fact more than one). We do this by obtaining a natural submodular function. First note that in a standard way, $\psi$ induces a function from the power set of $E$ into the power set of $S$. We abuse notation slightly and denote this function by $\psi$ also. That is, if $A$ is a subset of $E$ then $\psi(A)=\bigcup_{a \in A} \psi(a)$. In particular $\psi(\varnothing)=\varnothing$.

Now let $k$ be an arbitrary integer and let $\mu_{k}: 2^{E} \rightarrow Z$ be defined by $\mu_{k}(A)=$ $r_{M}(\psi(A))-k$ for all subsets $A \subseteq E$. That $\mu_{k}$ is increasing and submodular is virtually a corollary of Crapo and Rota [5, Proposition 7.2]. A proof is given here since it is of central importance to this paper.

Proposition 4.1. The function $\mu_{k}$ is increasing and submodular. 
Proof. Since the rank function of $M$ is increasing it follows immediately that $\mu_{k}$ is.

Elementary set theory establishes that $\psi(A \cup B)=\psi(A) \cup \psi(B)$ and that $\psi(A \cap B) \subseteq \psi(A) \cap \psi(B)$. Therefore

$$
\begin{aligned}
\mu_{k}(A \cup B)+\mu_{k}(A \cap B) & =r_{M}(\psi(A \cup B))+r_{M}(\psi(A \cap B))-2 k \\
& \leq r_{M}(\psi(A) \cup \psi(B))+r_{M}(\psi(A) \cap \psi(B))-2 k \\
& \leq r_{M}(\psi(A))+r_{M}(\psi(B))-2 k=\mu_{k}(A)+\mu_{k}(B)
\end{aligned}
$$

and $\mu_{k}$ is therefore submodular.

If $(Q, E, \psi)$ is a hypergraph on $Q$ then the matroid on $E$ induced by $\mu_{k}$ is denoted by $M_{k}(Q, E)$. The function $\psi$ while not mentioned in the notation is always understood. In situations where no danger of ambiguity exists we shall occasionally abbreviate the notation to $M_{k}$. If $N$ is a matroid on $E$ and there exists a function $\psi: E \rightarrow 2^{S}$ such that $N=M_{k}(Q, E)$ for some matroid $Q$ on $S$ and integer $k$, then $N$ is a $k$-induced matroid of $Q$. Clearly any matroid isomorphic to a $k$-induced matroid of $Q$ is also a $k$-induced matroid of $Q$.

Frequently in examples of interest it is the case that $Q$ is a free matroid. Here we shall blur the distinction between a matroid and its ground set and use the same symbol for both, typically " $V$ ".

The following proposition is an immediate consequence of Propositions 2.1 and 2.2 .

Proposition 4.2. Let $(Q, E, \psi)$ be a hypergraph on the matroid $Q$. Then

(i) the independent sets of $M_{k}(Q, E)$ are the subsets $I \subseteq E$ such that $r_{Q}\left(\psi\left(I^{\prime}\right)\right) \geq\left|I^{\prime}\right|+k$ for all nonempty subsets $I^{\prime} \subseteq I$; and

(ii) the circuits of $M_{k}(Q, E)$ are the minimal nonempty subsets $C \subseteq E$ with the property that $r_{Q}(\psi(C))<|C|+k$.

Corollary 4.3. Let $(V, E, \psi)$ be a hypergraph on the free matroid $V$. Then

(i) the independent sets of $M_{k}(V, E)$ are the subsets $I \subseteq E$ such that $\left|\psi\left(I^{\prime}\right)\right| \geq\left|I^{\prime}\right|+k$ for all nonempty subsets $I^{\prime} \subseteq I ;$ and

(ii) the circuits of $M_{k}(V, E)$ are the minimal nonempty subsets $C \subseteq E$ with the property that $|\psi(C)|<|C|+k$.

Proof. In a free matroid the cardinality of a subset is equal to its rank.

One reason for the study of $k$-induced matroids is that several important classes of matroids arise naturally in this way.

We first examine transversal matroids. Consider a 0 -induced matroid $M_{0}(V, E)$ of a free matroid $V$. Let $G$ be the bipartite graph with vertex set $V \cup E$ (we may assume that $V$ and $E$ are disjoint) and edge set as follows. An edge joins $v \in V$ to $e \in E$ exactly when $v \in \psi(e)$. As is well known (the original proof is in Edmonds and Fulkerson [10]) the subsets of $E$ which can be matched into $V$ are the independent sets of a matroid $M$ on $E$. A transversal 
matroid is one which is isomorphic to a matroid which can be obtained in such a way. For a subset $E^{\prime} \subseteq E, \psi\left(E^{\prime}\right)$ is just the set of neighbours of $E^{\prime}$ in $G$ (that is, the set of members of $V$ which are connected to a member of $E$ by an edge in $G$ ). It follows from the bipartite graph version of Hall's marriage theorem that a subset $I \subseteq E$ is independent in the transversal matroid $M$ if and only if $\left|\psi\left(I^{\prime}\right)\right| \geq\left|I^{\prime}\right|$ for all nonempty subsets $I^{\prime} \subseteq I$. But this is just the criterion for $I$ to be independent in $M_{0}(V, E)$ so $M=M_{0}(V, E)$. Since the construction is easily reversed it follows that the class of 0 -induced matroids of free matroids coincides with the class of transversal matroids. These observations are not new. The interested reader should consult Welsh [19, Chapters 7 and 8].

It is also the case that a matroid is a 0 -induced matroid of $(Q, E, \psi)$ if and only if it is induced from $Q$ in the sense of Welsh [19, Chapter 8.2].

We now consider matroids of graphs. Let $G=(V, E)$ be a graph. The incidence relation of $G$ induces a natural function $\psi$ from $E$ into the power set of $V$. If $e \in E$ then $\psi(e)=\{v \in V ; v$ is incident with $e\}$. If $|\psi(e)|=1$, then $e$ is a loop. In this paper we also allow the possibility that $|\psi(e)|=0$; that is, $e$ is not attached to any vertex. Such edges are the loose edges of Zaslavsky [23]. For graphs the above definition of $\psi$ shall remain in place throughout this paper. Now treat $V$ as a free matroid; then for any integer $k, M_{k}(V, E)$ is a well-defined matroid. This gives a class of matroids which one can naturally associate with $G$. Since $G=(V, E)$ it also makes sense to denote $M_{k}(V, E)$ by $M_{k}(G)$ and this we shall usually do for graphs.

What are these matroids like? Consider first the case $k=1$. By Corollary 2.4(i), a subset $I \subseteq E$ is independent in $M_{1}(G)$ if $\left|\psi\left(I^{\prime}\right)\right| \geq\left|I^{\prime}\right|+1$ for all nonempty subsets $I^{\prime} \subseteq I$. Elementary graph theory tells us that this is exactly the requirement for $I$ to be the edge set of a forest in $G$. That is, $I$ is independent in $M_{1}(G)$ if and only if $I$ is independent in $M(G)$, the cycle matroid of $G$ and we see that $M_{1}(G)$ is just the usual cycle matroid of $G$.

What about $k=2$ ? If $e \in E$, then $|\psi(e)| \leq 2$ and therefore $|\psi(e)|<$ $|\{e\}|+2$. So by Corollary 2.4(ii), $e$ is a circuit of $M_{2}(G)$. That is, $M_{2}(G)$ consists of loops. In fact for $k \geq 2, M_{k}(G)$ consists of loops only.

Of more interest is the case $k=0$. Since $M_{0}(G)$ is a 0 -induced matroid of a free matroid we see that $M_{0}(G)$ is transversal. In fact it follows, without difficulty, from results in [14] that $M_{0}(G)$ is precisely the bicircular matroid of $G$. Bicircular matroids of graphs are defined and studied in Matthews [14] and Simões-Pereira $[17,18]$. Recent interest centres on the role played by bicircular matroids in Zaslavsky's theory of bias matroids [24] (but see also [4]).

Matthews [14] notes that for $k<0, M_{k}(G)$ is the elongation (see [19, p. 60]) of the bicircular matroid, $M_{0}(G)$.

We now consider Dilworth truncations. If $\mathscr{F}_{k+1}$ is the set of all rank- $(k+1)$ flats of a matroid $Q$ on $E$ and $\psi$ is the identity function then $M_{k}\left(Q, \mathscr{F}_{k+1}\right)$ 

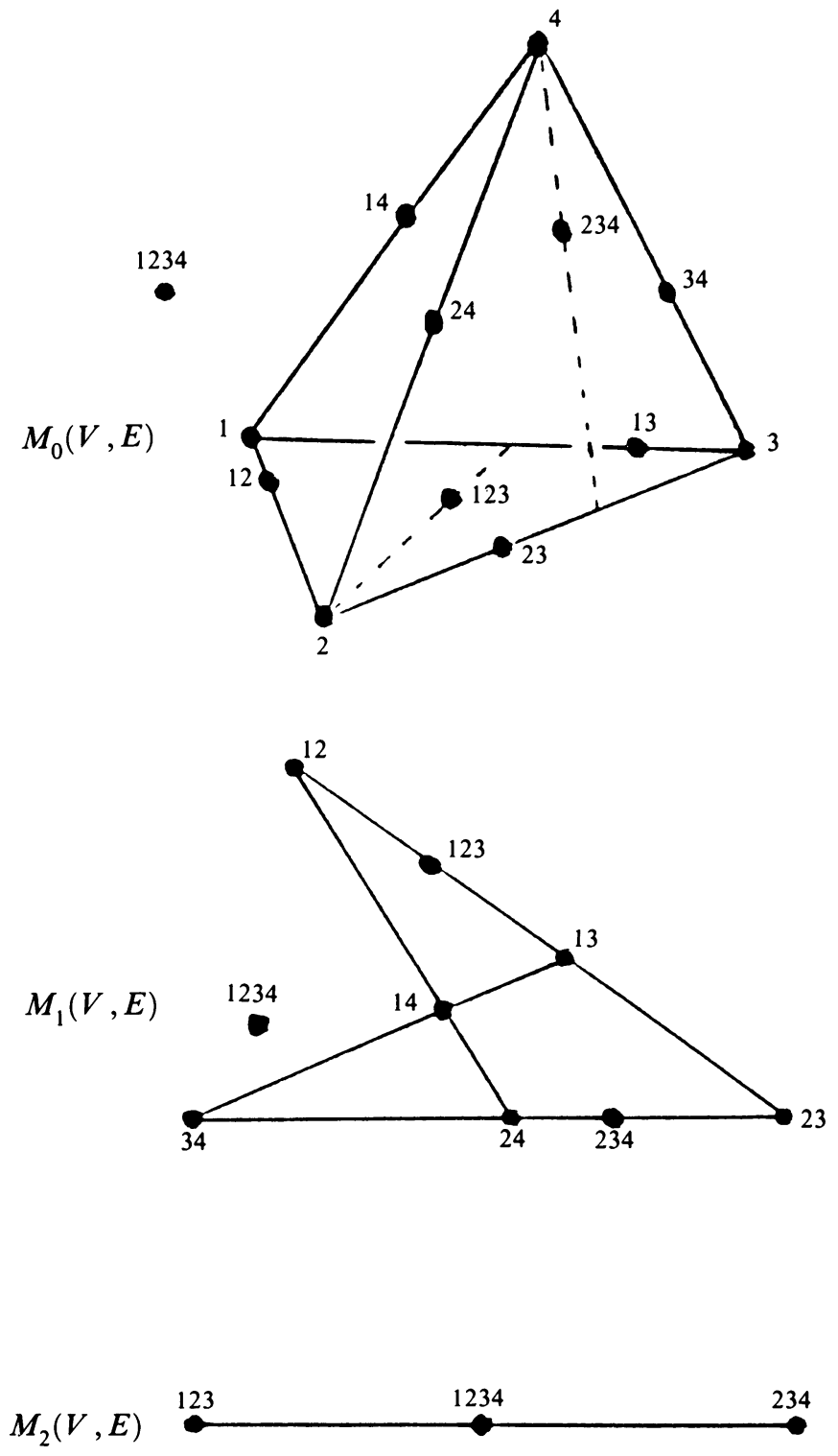

$M_{3}(V, E)$

Points not indicated are loops 
is the $k$ th Dilworth truncation of $Q$. (This follows immediately from the definition of the Dilworth truncation.) Brylawski [2], Crapo and Rota [5, Chapter 7] and Mason [13] all contain interesting discussions on Dilworth truncations. While we do not use results on Dilworth truncations in this paper it is the case that the theory of $k$-induced matroids is just the theory of generalised Dilworth truncations.

Little of this section is new (except perhaps the presentation). Nonetheless it is my belief that many, apparently distinct, theorems in graph theory, transversal theory and Dilworth truncations have natural and unified common generalisations in the theory of $k$-induced matroids (an example of this is provided by Theorem 6.2 below). Also, since these matroids are all induced by a submodular function which is in some sense canonical (namely $\mu_{k}$ ) the study of $\mu_{k}$-lifts of $k$-induced matroids would seem worthwhile. We shall presently see that in some cases this is certainly so. But first we provide an example to illustrate some of the concepts in this section.

Let $V$ be the free matroid on $\{1,2,3,4\}, E$ be the set $\{1,2,3,4,12,23$, $34,14,13,24,123,234,1234\}$ and $\psi: E \rightarrow 2^{V}$ be the natural map. That is, $\psi(i j)=\{i, j\}$ etc. Then Figure 1 gives Euclidean representations of $M_{0}(V, E)$, $M_{1}(V, E), M_{2}(V, E)$ and $M_{3}(V, E)$.

\section{BIAS MATROIDS}

Let $\mathscr{B}$ be a linear class of circuits of the $k$-induced matroid $M_{k}(Q, E)$. Denote the $\mu_{k}$-lift of $M_{k}(Q, E)$ induced by $\mathscr{B}$ by $M_{k}^{\mathscr{B}}(Q, E)$. It follows immediately from the definition of $\mu_{k}$ that $\mu_{k}+1=\mu_{k-1}$. Therefore $M_{k}^{\mathscr{B}}(Q, E)$ is a matroid intermediate between $M_{k}(Q, E)$ and $M_{k-1}(Q, E)$ in the weak map order.

A case of particular interest occurs for 1-induced matroids of graphs. If $M_{1}(G)$ is the 1-induced matroid of the graph $G$ then $M_{1}^{B}(G)$ is a matroid intermediate between $M_{0}(G)$ and $M_{1}(G)$ in the weak map order. Since $M_{1}(G)$ is the cycle matroid of the graph $G$ and $M_{0}(G)$ is the bicircular matroid of $G$ we obtain immediately the following description of $M_{1}^{B}(G)$.

Proposition 5.1. Let $\mathscr{B}$ be a linear class of circuits of the cycle matroid of a graph $G$. Then a subset $C$ of edges of $G$ is a circuit of $M_{1}^{B}(G)$ if and only if either $C \in \mathscr{B}$ or $C$ is a circuit of the bicircular matroid of $G$ containing no member of $\mathscr{B}$.

We now show that $M_{1}^{\beta}(G)$ is the bias matroid of a biased graph in the sense of Zaslavsky [24]. A theta graph is a subdivision of a connected graph on two vertices with three parallel edges; that is, one which consists of three paths meeting at their endpoints. Zaslavsky (see for example [23, 24]) defines a set $\mathscr{B}$ of circuits of $G$ to be a linear class if no theta subgraph of $G$ contains exactly two members of $\mathscr{B}$. It is not hard to show that a connected graph $G$ is a theta graph if and only if the edge set of $G$ is the union of a modular pair of 
distinct, nondisjoint circuits of the cycle matroid of $G$. It then follows, without difficulty, that a set of circuits of $G$ is a linear class (in the sense of Zaslavsky) if and only if their edge sets form a linear class (in the sense of this paper) in the cycle matroid of $G$.

A biased graph is a graph together with a linear class of circuits. That $M_{1}^{\mathscr{B}}(G)$ is the bias matroid of the biased graph $G$ with linear class $\mathscr{B}$ of circuits follows routinely from the above discussion and Zaslavsky's characterisation [24, §2] of this matroid.

The importance of such bias matroids is difficult to overestimate. We remind the reader again that Dowling group geometries are examples of bias matroids. In general, the bias matroid of a biased graph is different from the elementary lift of the cycle matroid of the graph induced by the same linear class of circuits (see, for example, $[28 ; 24, \S 4]$ ). (In our language, if $\mathscr{B}$ is a linear class of circuits of $M(G)$ then the $\mu_{1}$-lift of $M(G)$ induced by $\mathscr{B}$ is in general not isomorphic to the $r$-lift of $M(G)$ induced by $\mathscr{B}$.) Furthermore it appears that bias matroids of graphs form a more interesting class than elementary lifts of graphic matroids. But perhaps this is not surprising; the function $\mu_{1}$ is derived from the incidence relation of the graph and is therefore a most natural submodular function. For other classes of matroids induced by "natural" submodular functions it may be the case that the $\mu$-lifts associated with these submodular functions form interesting classes. In particular $\mu_{k}$-lifts of more general $k$-induced matroids could well reward study.

\section{MINORS}

In this section we characterise minors of $k$-induced matroids and minors of $\mu_{k}$-lifts of $k$-induced matroids. As usual let $(Q, E, \psi)$ be a hypergraph on the matroid $Q$ and consider $M_{k}(Q, E)$.

To characterise minors of $M_{k}(Q, E)$ it suffices to characterise matroids obtained by deleting or contracting single points. Deletion causes no difficulty since it is immediate that if $e \in E$, then $M_{k}(Q, E) \backslash e=M_{k}(Q, E \backslash e)$. (Note that strictly speaking $M_{k}(Q, E \backslash e)$ is not well defined unless a function from $E \backslash e$ into the power set of the ground set of $Q$ is specified. This function is here (and in any other unexplained context) just the appropriate restriction of $\psi$.) Contracting a single point is not quite so straightforward. We need some background.

If $A$ is a set of points of a matroid $M$, then the principal truncation of $M$ at $A$, denoted $T_{A}(M)$, is the elementary quotient of $M$ induced by the modular cut consisting of all flats of $M$ containing $A$. One routinely obtains:

Proposition 6.1. If $M$ is a matroid on $E$ and $A$ is a subset of $E$ with $r_{M}(A)>$ 1 , then

(i) a subset $I \subseteq E$ is independent in $T_{A}(M)$ if and only if $I$ is independent in $M$ and $\mathrm{cl}_{M}(I)$ does not contain $A$; 
(ii) for a subset $B \subseteq E$,

$$
r_{T_{A}(M)}(B)= \begin{cases}r_{M}(B) & \text { if } A \nsubseteq \mathrm{cl}_{M}(B), \\ r_{M}(B)-1 & \text { if } A \subseteq \mathrm{cl}_{M}(B) .\end{cases}
$$

We can now examine single point contractions of $M_{k}(Q, E)$. If $e \in E$ is a loop of $M_{k}(Q, E)$, then $M_{k}(Q, E) / e=M_{k}(Q, E) \backslash e=M_{k}(Q, E \backslash e)$ so it suffices to consider the case when $e$ is not a loop.

Theorem 6.2. If an element $e$ of $E$ is not a loop of $M_{k}(Q, E)$ and if $r_{Q}(\psi(e))>$ 0 , then $M_{k}(Q, E) / e=M_{k}\left(T_{\psi(e)}(Q), E \backslash e\right)$.

Proof. Since $e$ is not a loop of $M$, the independent sets of $M_{k}(Q, E) / e$ are the subsets $I \subseteq E$ such that $I \cup e$ is independent in $M_{k}(Q, E)$. We show that the independent sets of $M_{k}(Q, E) / e$ and $M_{k}\left(T_{\psi(e)}(Q), E \backslash e\right)$ coincide. Certainly the empty set is independent in both matroids, so let us begin by assuming that $I$ is a nonempty independent set of $M_{k}(Q, E) / e$ and let $I^{\prime}$ be a nonempty subset of $I$. Then $I^{\prime} \cup e$ is independent in $M_{k}(Q, E)$, so $r_{Q}\left(\psi\left(I^{\prime} \cup e\right)\right) \geq\left|I^{\prime} \cup e\right|+k$ and also $r_{Q}\left(\psi\left(I^{\prime}\right)\right) \geq\left|I^{\prime}\right|+k$. If $\psi(e) \subseteq \mathrm{cl}_{Q}\left(\psi\left(I^{\prime}\right)\right)$, then $r_{Q}\left(\psi\left(I^{\prime} \cup e\right)\right)=r_{Q}\left(\psi\left(I^{\prime}\right)\right)$ and by Proposition 6.1(ii), $r_{T_{\psi(e)}(Q)}\left(\psi\left(I^{\prime}\right)\right)=r_{Q}\left(\psi\left(I^{\prime}\right)\right)-1$. Hence

$$
r_{T_{\psi(e)}(Q)}\left(\psi\left(I^{\prime}\right)\right)=r_{Q}\left(\psi\left(I^{\prime} \cup e\right)\right)-1 \geq\left|I^{\prime} \cup e\right|+k-1=\left|I^{\prime}\right|+k .
$$

If $\psi(e) \nsubseteq \mathrm{cl}_{Q}\left(\psi\left(I^{\prime}\right)\right)$, then $r_{T_{\psi(e)}(Q)}\left(\psi\left(I^{\prime}\right)\right)=r_{Q}\left(\psi\left(I^{\prime}\right)\right) \geq\left|I^{\prime}\right|+k$. In either case $r_{T_{\psi(e)}(Q)}\left(\psi\left(I^{\prime}\right)\right) \geq\left|I^{\prime}\right|+k$ and since this holds for all nonempty subsets $I^{\prime} \subseteq I$, $I$ is independent in $M_{k}\left(T_{\psi(e)}(Q), E \backslash e\right)$.

Conversely assume that $I$ is independent in $T_{\psi(e)}(Q)$ and let $I^{\prime}$ be a nonempty subset of $I$. Note that $r_{T_{\psi(e)}(Q)}\left(\psi\left(I^{\prime}\right)\right) \geq\left|I^{\prime}\right|+k$. If $\psi(e) \subseteq \mathrm{cl}_{Q}\left(\psi\left(I^{\prime}\right)\right)$, then

$$
\begin{aligned}
r_{Q}\left(\psi\left(I^{\prime} \cup e\right)\right) & =r_{Q}\left(\psi\left(I^{\prime}\right)\right)=r_{T_{\psi(e)}(Q)}\left(\psi\left(I^{\prime}\right)\right)+1 \\
& \geq\left|I^{\prime}\right|+k+1=\left|I^{\prime} \cup e\right|+k
\end{aligned}
$$

and if $\psi(e) \nsubseteq \mathrm{cl}_{Q}\left(\psi\left(I^{\prime}\right)\right)$, then

$$
\begin{aligned}
r_{Q}\left(\psi\left(I^{\prime} \cup e\right)\right) & \geq r_{\dot{Q}}\left(\psi\left(I^{\prime}\right)\right)+1=r_{T_{\psi(e)}(Q)}\left(\psi\left(I^{\prime}\right)\right)+1 \\
& \geq\left|I^{\prime}\right|+k+1=\left|I^{\prime} \cup e\right|+k .
\end{aligned}
$$

In either case, $r_{Q}\left(\psi\left(I^{\prime} \cup e\right)\right) \geq\left|I^{\prime} \cup e\right|+k$. We must also note that $r_{Q}\left(\psi\left(I^{\prime}\right)\right) \geq$ $r_{T_{\psi(e)}(Q)}\left(\psi\left(I^{\prime}\right)\right) \geq\left|I^{\prime}\right|+k$. This suffices to show that $I \cup e$ is independent in $M_{k}(Q, E)$ and therefore that $I$ is independent in $M_{k}(Q, E) / e$.

Since $M_{k}(Q, E) / e$ and $M_{k}\left(T_{\psi(e)}(Q), E \backslash e\right)$ share common ground sets, the theorem is proved.

No doubt a more general theorem along the lines of [22, Theorem 3.3] could be proved but Theorem 6.2 suffices here. 
Let $\mathscr{Q}$ be a class of matroids and $k$ be a fixed integer. Denote the class of $k$-induced matroids of members of $\mathscr{Q}$ by $\mathscr{M}_{k}(\mathscr{Q})$. That is, $M \in \mathscr{M}_{k}(\mathscr{Q})$ if and only if $M=M_{k}(Q, E)$ for some $Q \in \mathscr{Q}$. It is of interest to ask when $\mathscr{M}_{k}(\mathscr{Q})$ will be minor-closed. It is easily seen that $\mathscr{M}_{k}(\mathscr{Q})$ is closed under restrictions for any class $\mathscr{Q}$ so $\mathscr{M}_{k}(\mathscr{Q})$ is minor-closed whenever $\mathscr{M}_{k}(\mathscr{Q})$ is closed under contractions. In all but the most trivial cases $\mathscr{M}_{k}(\mathscr{Q})$ is not closed under contractions if $k<0$. For say $M \in \mathscr{M}_{k}(\mathscr{Q})$ is not free, then $M$ contains a loop as a minor. But it is easily seen that for $k<0$, all members of $\mathscr{M}_{k}(\mathscr{Q})$ are loopless. The problem is due to the fact that for such values of $k$ it may well be the case that there exists a nonloop $e$ of $M_{k}(Q, E)$ with $r_{Q}(\psi(e))=0$. This does not happen when $k \geq 0$ and we have:

Proposition 6.3. Let $k$ be a nonnegative integer and $\mathscr{Q}$ be a class of matroids. Assume that for any member $Q$ of $\mathscr{Q}, T_{S}(Q) \in \mathscr{Q}$ whenever $S$ is a subset of the ground set of $Q$ with $r_{Q}(S) \geq k+1$. Then $\mathscr{M}_{k}(\mathscr{Q})$ is minor-closed.

Proof. Say $M_{k}(Q, E) \in \mathscr{M}_{k}(\mathscr{Q})$ and that the element $e \in E$ is not a loop of $M_{k}(Q, E)$. Then $r_{Q}(\psi(e)) \geq r_{M_{k}(Q, E)}(e)+k=k+1 \geq 1$. So the conditions of Theorem 6.2 are satisfied and therefore $M_{k}(Q, E) / e=M_{k}\left(T_{\psi(e)}(Q), E \backslash e\right)$. But $r_{Q}(\psi(e)) \geq k+1$ so $T_{\psi(e)}(Q) \in \mathscr{Q}$ and therefore $M_{k}\left(T_{\psi(e)}(Q), E \backslash e\right) \in \mathscr{M}_{k}(\mathscr{Q})$. This suffices, in the light of the previous discussion, to establish that $\mathscr{M}_{k}(\mathscr{Q})$ is minor-closed.

As a special case we have

Corollary 6.4. $\mathscr{M}_{k}(\mathscr{Q})$ is minor-closed whenever $k \geq 0$ and $\mathscr{Q}$ is closed under principal truncations.

This leads to some examples.

A strict gammoid is a matroid whose dual is transversal. Dowling and Kelly [9] show that the class of strict gammoids is also the class of matroids obtained by successively taking principal truncations of free matroids (albeit using a slightly different terminology). Let $\mathscr{T}^{*}$ denote the class of strict gammoids. As an immediate corollary of Corollary 6.4 we obtain

Corollary 6.5. The class $\mathscr{M}_{k}\left(\mathscr{T}^{*}\right)$ is minor-closed for any integer $k \geq 0$.

In particular the class $\mathscr{M}_{0}\left(\mathscr{T}^{*}\right)$ is minor-closed. This is just the class of gammoids; that is, the class of minors of transversal matroids.

Let $\mathscr{Q}_{p}$ denote the class of matroids representable over a field of characteristic $p$. Brylawski [3, Proposition 7.4.10] shows that $\mathscr{Q}_{p}$ is closed under principal truncations and therefore we have

Corollary 6.6. The class $\mathscr{M}_{k}\left(\mathscr{Q}_{p}\right)$ is minor-closed for any integer $k \geq 0$.

Brylawski [2, Theorem 3.1] also shows that whenever a matroid is representable over a field of characteristic $p$, then the Dilworth truncation of $M$ is 
representable over a field of characteristic $p$. (In fact this is a result of Mason $[13, \S 2.4]$ but Mason's proof is not rigorous.) As a generalisation of this theorem we conjecture that $\mathscr{M}_{k}\left(\mathscr{Q}_{p}\right) \subseteq \mathscr{Q}_{p}$.

Of course certain interesting subclasses of $\mathscr{M}_{k}(\mathscr{Q})$ may be minor-closed even when $\mathscr{M}_{k}(\mathscr{Q})$ is not. For example, if $\mathscr{V}$ is the class of free matroids, then $\mathscr{M}_{1}(\mathscr{V})$ is not minor-closed but the subclass of graphic matroids is. This follows readily from the fact that whenever $V$ is a free matroid and a subset $S \subseteq V$ has $|S| \leq 2$, then the simplification of $T_{S}(V)$ is free. Considering the simplification of $T_{S}(V)$ here corresponds to coalescing vertices in contracting an edge from a graph.

We now consider minors of $\mu_{k}$-lifts of $k$-induced matroids. If $e$ is a nonloop of the matroid $M$ on $E$, then as is very well known, the circuits of $M / e$ are the minimal subsets of $E$ of the form $\{C \backslash e: C$ is a circuit of $M\}$. A routine consequence of this fact is

Proposition 6.7. If $e$ is a nonloop of the matroid $M$ on $E$, then a subset $C^{\prime} \subseteq$ $E \backslash e$ is a circuit of $M / e$ if and only if either

(i) $C^{\prime} \cup e$ is a circuit of $M$, or

(ii) $C^{\prime}$ is a circuit of $M$ and $e \notin \mathrm{cl}_{M}\left(C^{\prime}\right)$.

Now let $\mathscr{B}$ be a linear class of circuits of $M$. For $e \in E$ we define $\mathscr{B} \backslash e$ to be the subset of $\mathscr{B}$ consisting of those members of $\mathscr{B}$ which do not contain $e$. With the added condition that $e$ is not a loop of $M$ we define $\mathscr{B} / e$ to be the set consisting of those members of the set $\{C \backslash e: C \in \mathscr{B}\}$ which are circuits of $M / e$. Note that it is quite possible for a minimal member of the set $\{C \backslash e: C \in \mathscr{B}\}$ to be a noncircuit of $M / e$.

Lemma 6.8. (i) $\mathscr{B} \backslash e$ is a linear class of circuits of $M \backslash e$.

(ii) If $e$ is a nonloop of $M$, then $\mathscr{B} / e$ is a linear class of circuits of $M / e$.

Proof. Part (i) is trivial so consider (ii). By definition, the members of $\mathscr{B} / e$ are all circuits of $M / e$ so it suffices to show that if distinct circuits $C_{1}$ and $C_{2}$ are a modular pair (in $M / e$ ) of members of $\mathscr{B} / e$, then every circuit of $C$ of $M / e$ contained in $C_{1} \cup C_{2}$ belongs to $\mathscr{B} / e$. Assume therefore that $C_{1}$ and $C_{2}$ are such a pair of circuits. There are two cases to consider.

Assume that $C_{1} \cup e$ is a circuit of $M$. Either $C_{2}$ or $C_{2} \cup e$ is a circuit $C_{2}^{\prime}$ of $M$. Now $r_{M}\left(C_{1} \cup C_{2} \cup e\right)=r_{M / e}\left(C_{1} \cup C_{2}\right)+1$ and by Proposition 3.2, $r_{M / e}\left(C_{1} \cup C_{2}\right)=\left|C_{1} \cup C_{2}\right|-2$. Therefore $r_{M}\left(C_{1} \cup C_{2} \cup e\right)=\left|C_{1} \cup C_{2} \cup e\right|-2$ so $C_{1} \cup e$ and $C_{2}^{\prime}$ are a modular pair of circuits of $M$. Since both $C_{1} \cup e$ and $C_{2}^{\prime}$ belong to $\mathscr{B}$ it follows that every circuit of $M$ contained in $C_{1} \cup C_{2} \cup e$ belongs to $\mathscr{B}$. If a subset $C \subseteq C_{1} \cup C_{2}$ is a circuit of $M / e$, then either $C$ or $C \cup e$ is a circuit $C^{\prime}$ of $M$. In either case $C^{\prime} \in \mathscr{B}$ so $C \in \mathscr{B} / e$.

Assume that both $C_{1}$ and $C_{2}$ are circuits of $M$. Then

$$
r_{M}\left(C_{1} \cup C_{2}\right) \geq r_{M / c}\left(C_{1} \cup C_{2}\right)=\left|C_{1} \cup C_{2}\right|-2 .
$$


But $r_{M}\left(C_{1} \cup C_{2}\right) \leq\left|C_{1} \cup C_{2}\right|-2$ for any pair of distinct circuits so the above inequality is in fact an equality. This shows that $C_{1}$ and $C_{2}$ are a modular pair of circuits in $M$. But also, since $r_{M}\left(C_{1} \cup C_{2}\right)=r_{M / e}\left(C_{1} \cup C_{2}\right)$, we have shown that $e \notin \mathrm{cl}_{M}\left(C_{1} \cup C_{2}\right)$. Since $C_{1}$ and $C_{2}$ are a modular pair of circuits of $M$ and both belong to $\mathscr{B}$, any circuit of $M$ contained in $C_{1} \cup C_{2}$ also belongs to $\mathscr{B}$. Now if $C$ is a circuit of $M / e$ contained in $C_{1} \cup C_{2}$ then $\mathrm{cl}_{M}(C) \subseteq \mathrm{cl}_{M}\left(C_{1} \cup C_{2}\right)$ so $e \notin \mathrm{cl}_{M}(C)$ and it follows that $C$ is a circuit of $M$. Therefore $C \in \mathscr{B}$ and also $C \in \mathscr{B} / e$.

In either case, if $C_{1}$ and $C_{2}$ are a modular pair of members of $\mathscr{B} / e$, then every circuit $C$ of $M / e$ contained in $C_{1} \cup C_{2}$ belongs to $\mathscr{B} / e$ and the theorem is proved.

Recall that if $\mathscr{B}$ is a linear class of circuits of $M_{k}(Q, E)$ then the $\mu_{k}$-lift of $M_{k}(Q, E)$ induced by $\mathscr{B}$ is denoted by $M_{k}^{\mathscr{B}}(Q, E)$. We are now able to characterise single-element deletions and contractions of $M_{k}^{\mathscr{B}}(Q, E)$.

Theorem 6.9. If $\mathscr{B}$ is a linear class of circuits of $M_{k}(Q, E)$ and $e$ is an element of $E$, then

(i) $M_{k}^{\mathscr{B}}(Q, E) \backslash e=M_{k}^{\mathscr{B} \backslash e}(Q, E \backslash e)$, and

(ii) if $e$ is a nonloop of $M_{k}(Q, E)$ and $r_{Q}(\psi(e))>0$, then $M_{k}^{\mathscr{B}}(Q, E) / e=$ $M_{k}^{\mathscr{B} / e}\left(T_{\psi(e)}(Q), E \backslash e\right)$.

Proof. Consider (i). Since $M_{k}(Q, E) \backslash e=M_{k}(Q, E \backslash e)$ it follows from Lemma 6.7 (i) that $\mathscr{B} \backslash e$ is a linear class of circuits of $M_{k}(Q, E \backslash e)$ and therefore $M_{k}^{\mathscr{B} \backslash e}(Q, E \backslash e)$ is well defined. Now a subset $D \subseteq E$ is dependent in $M_{k}^{\mathscr{B} \backslash e}(Q, E \backslash e)$ if and only if either $D$ contains a circuit of $M_{k-1}(Q, E \backslash e)$ or $D$ contains a member of $\mathscr{B} \backslash e$. But circuits of $M_{k-1}(Q, E \backslash e)$ are just circuits of $M_{k-1}(Q, E)$ not containing $e$ and members of $\mathscr{B} \backslash e$ are just members of $\mathscr{B}$ not containing $e$. So $D$ is dependent in $M_{k}^{\mathscr{B} \backslash e}(Q, E \backslash e)$ if and only if $D$ is dependent in $M_{k}^{\mathscr{B}}(Q, E) \backslash e$ and the two matroids are therefore equal.

Now consider (ii). By Theorem 6.1, $M_{k}(Q, E) / e=M_{k}\left(T_{\psi(e)}(M), E \backslash e\right)$ and by Proposition 6.7(ii), $\mathscr{B} / e$ is a linear class of circuits of $M_{k}(Q, E) / e$ so $M_{k}^{\mathscr{B} / e}\left(T_{\psi(e)}(Q), E \backslash e\right)$ is well defined. Now a subset $D \subseteq E$ is dependent in $M_{k}^{\mathscr{B}}(Q, E)$ if and only if either $D$ contains a circuit of $M_{k-1}(Q, E)$ or $D$ contains a member of $\mathscr{B}$. Since $e$ is a nonloop of $M_{k}(Q, E), D$ is dependent in $M_{k}^{\mathscr{B}}(Q, E) / e$ if and only if $D \cup e$ is dependent in $M_{k}^{\mathscr{B}}(Q, E)$. That is, $D$ is dependent in $M_{k}^{\mathscr{B}}(Q, E) / e$ if and only if either $D \cup e$ contains a circuit of $M_{k-1}(Q, E)$ or $D \cup e$ contains a member of $\mathscr{B}$. If $D \cup e$ contains a member of $\mathscr{B}$ but does not contain a circuit of $M_{k-1}(Q, E)$, then $D$ contains a minimal member $D^{\prime}$ of the set $\{C \backslash e: C \in \mathscr{B}\}$. But $D^{\prime}$ must be a circuit of $M_{k}^{\mathscr{B}}(Q, E) / e$ (for otherwise $D \cup e$ would contain a circuit of $M_{k-1}(Q, E)$ ). 
Therefore $D$ contains a member of $\mathscr{B} / e$. Conversely if $D$ contains a member of $\mathscr{B} / e$, then $D \cup e$ certainly contains a member of $\mathscr{B}$. Moreover a subset $D \subseteq E$ is dependent in $M_{k-1}\left(T_{\psi(e)}(Q), E \backslash e\right)=M_{k-1}(Q, E) / e$ if and only if $D \cup e$ is dependent in $M_{k-1}(Q, E)$. Therefore $D$ is dependent in $M_{k}^{\not B}(Q, E) / e$ if and only if either $D$ contains a circuit of $M_{k-1}\left(T_{\psi(e)}(Q), E \backslash e\right)$ or $D$ contains a member of $\mathscr{B} / e$; that is, if and only if $D$ is dependent in $M_{k}^{\mathscr{B} / e}\left(T_{\psi(e)}(Q), E \backslash e\right)$. Therefore

$$
M_{k}^{\mathscr{B} / e}\left(T_{\psi(e)}(Q), E \backslash e\right)=M_{k}^{\mathscr{B}}(Q, E) / e .
$$

\section{REFERENCES}

1. C. Berge, Graphs and hypergraphs, North-Holland, Amsterdam, 1976.

2. T. Brylawski, Coordinatizing the Dilworth truncation, in Matroid Theory (L. Lovász and A. Recski, eds.), Colloq. Math. Soc. János Bolyai, no. 40, North-Holland, Amsterdam, 1985.

3. T. Brylawski, Constructions, Chapter 7 in [20].

4. C. R. Coullard, J. G. del Greco and D. K. Wagner, Representations of bicircular matroids, preprint.

5. H. H. Crapo and G.-C. Rota, On the foundations of combinatorial theory: Combinatorial geometries, M.I.T. Press, Cambridge, Mass., 1970.

6. J. E. Dawson, Balanced sets in an independence structure induced by a submodular function, J. Math. Anal. Appl. 95 (1983), 214-222.

7. P. Doubilet, G.-C. Rota and R. Stanley, On the foundations of combinatorial theory (IV): The idea of generating functions, Proc. Sixth Berkeley Sympos. on Mathematical Statistics and Probability, Berkeley, 1970/1971, vol. 11, Probability Theory, Univ. of California Press, Berkeley, Calif., 1972, pp. 276-318.

8. T. A. Dowling, A class of geometric lattices based on finite groups, J. Combin. Theory Ser. B 14 (1973), 61-86.

9. T. A. Dowling and D. G. Kelly, Elementary strong maps and transversal geometries, Discrete Math. 7 (1974), 209-224.

10. J. Edmunds and D. R. Fulkerson, Transversals and matroid partitions, J. Res. Nat. Bur. Standards B 69 (1965), 147-153.

11. J. Kahn and J. P. S. Kung, Varieties of combinatorial geometries, Trans. Amer. Math. Soc. 271 (1982), 485-499.

12. C. Lintzeris, Economical presentation of matroids by functions, Ph.D. thesis, University of Tasmania, 1987.

13. J. H. Mason, Matroids as the study of geometrical configurations, in Higher Combinatorics (M. Aigner, ed.), Reidel, Dordrecht, 1977.

14. L. R. Matthews, Bicircular matroids, Quart. J. Math. Oxford Ser. (2) 28 (1977), 213-228.

15. J. G. Oxley, A characterization of the ternary matroids with no $M\left(K_{4}\right)-$ minor, J. Combin. Theory Ser. B 42 (1987), 212-249.

16. J. S. Pym and H. Perfect, Submodular functions and independence structures, J. Math. Anal. Appl. 30 (1970), 1-31.

17. J. M. S. Simòes-Pereira, On subgraphs as matroid cells, Math. Z. 127 (1972). 315-322.

18. _ On matroids on edge sets of graphs with connected subgraphs as circuits. II, Discrete Math. 12 (1975), 55-78.

19. D. J. A. Welsh, Matroid theory, London Math. Soc. Monographs, No. 8, Academic Press, New York, 1976. 
20. N. L. White, ed., Theory of matroids (Encyclopedia of Math. and Its Applications), Cambridge Univ. Press, Cambridge, 1986.

21. G. P. Whittle, Dowling group geometries and the critical problem, J. Combin. Theory Ser. B (to appear).

22. —_ Quotients of Dilworth truncations, J. Combin. Theory Ser. B (to appear).

23. T. Zaslavsky, Biased graphs. I. Bias, balance and gains, preprint.

24. _ Biased graphs. II. The three matroids, preprint.

25. __ Biased graphs. III. Chromatic and dichromatic invariants, preprint.

26. __ Biased graphs. IV. Geometric realizations, preprint.

27. __ Biased graphs. V. Universal and topological gains, preprint.

28. __ Biased graphs whose matroids are special binary matroids, preprint.

Department of Mathematics, University of Tasmania, G.P.O. Box 252C, Hobart, TasMANia 7001, AUSTRALIA 